\title{
Grasping force control of a tendon-driven prosthetic finger based on force estimation using motor current signals
}

\author{
Wendi Zhuo ${ }^{1,2}$, Yi Zhang ${ }^{1,2}$ and Hua Deng ${ }^{1,2, a}$ \\ ${ }^{1}$ State key laboratory of High-Performance Complex Manufacturing; \\ ${ }^{2}$ School of Mechanical \& Electrical Engineering, Central South University, 410083 Changsha, China
}

\begin{abstract}
A force estimation model using motor current signals was deduced in this paper for a tendon-driven prosthetic finger grasping objects with its distal phalanx. Models of the prosthetic finger were first established. As driving moment of each joint could be calculated form motor current, stable grasping force of the finger could be calculated by its statics mechanic model, that is, the grasping force is estimated using motor current signals. Then a PID controller based on the estimation method was designed with the estimated force as its force feedback signal. Based on the dynamics model of the prosthetic finger and a DC motor model, the estimation model and the PID controller were simulated in MATLAB, whose results indicated that the proposed grasping force estimation and control methods are effective.
\end{abstract}

Keywords: prosthetic finger; tendon-driven; force estimation using motor current; grasping force control; PID control algorithm.

\section{Introduction}

Although bionic prosthetic hand technology advances rapidly, bionic prostheses are still inferior replacements of biological entities. There are still a lot of difficulties to overcome to completely imitate human hands or even beyond its inherent functions. An ongoing challenge for prosthetic hand developers is how to copy the feeling of human hands. The prosthetic hand needs interpretation of their own tactile information to adjust its actions. For example, the grasping force information can ensure that users will not damage the grasped object because of a tight grip. This information is required for direct control of the device, and can be obtained through a low-end control loop. As a result, the user of the prosthetic hand need not rely on vision information to control the magnitude of grasping force [1].

To obtain tactile feedback information, force sensors should cover all the possible grip positions [2], increasing the weight and cost of the prosthetic hand obviously. As long as the prosthetic hands grasp an object, the reaction force of the object to the finger must result in a change of the driving motor torque, and the change will then lead to changes in motor current, regardless of the contact position between the object and the finger. Thus, a grasping force estimation method of prosthetic hands by detecting the motor current signals is proposed. If different current estimation models are established for every possible contact positions, the force sensor measurement can be totally replaced by current detection. Then grasping force of prosthetic hands could be controlled based on the force estimates instead of

\footnotetext{
${ }^{\text {a }}$ Corresponding author : hdeng@csu.edu.cn
} 
force sensor readings, which will lower the cost and weight of the prosthetic hand and make them more convenient to use.

Under-actuated coupling prosthetic hands have significantly reduced the number of prosthetic hand drivers, lowering the control difficulty of prosthetic hands and also ensuring a certain adaptive grasping ability of their fingers [3]. Under-actuated coupling prosthetic hands have a relatively simple structure-the tendon-driven structure, which adopts a kind of driving mode that uses the tendon to transmit power and motion [4]. The structure of the tendon-driven prosthetic hand is simple and compact with small size and light weight, making it easily in line with the shape, size and weight of human hands. Because this driving pattern is similar to the tendon-driven mechanism in an organism, it has been widely used in the field of bionic robots and artificial limbs [5, 6].

In this paper, the models of a tendon-driven prosthetic finger were established first, and then the force estimation model using motor current signals was established based on them. Next, the estimation method was applied to a PID grasping force control system of the finger. Finally, the effectiveness of the proposed force estimation and control methods are validated by the MATLAB simulations.

\section{Prosthetic hand models}

A tendon-driven prosthetic finger, whose main component parameters - drive pulley radius, spring stiffness coefficients and spring preload force of each phalange, are designed, is shown in Figure 1, The prosthetic finger is composed by three phalanxes, and has three joints and one driver, whose structure diagram is shown in Figure 2. The tendon rope driven by a DC motor through a speed reducing mechanism drives the finger joints to rotate. When the motor stops working, the finger is locked by the worm and gear. When the finger needs to be reset, the motor reverses and relaxes the tendon, so the reverse force is applied by return springs to reset the joints at the same time. Grip and reset actions of the prosthetic finger are thus achieved. The double pulley structure, which splits the tendon driving rope into two: $N_{1}$ and $N_{2}$, is applied in the middle phalanx to distribute the torque of each joint reasonably. $N_{1}$, which is connected with the motor, is used as a driving rope running through the proximal joint and the middle joint. $N_{2}$ runs through the middle joint and the distal joint. Each pulley and the corresponding link rod are regarded as a whole, and their mass and moment of inertia are integrated. The simplified prosthetic finger is composed of three link rods and three springs, and tensile force of the rope is denoted as $F$. The external force of proximal, middle and distal phalanxes is $F_{1}, F_{2}$ and $F_{3}$ respectively, which are $S_{1}, S_{2}$ and $S_{3}$ away from each joint. Before the finger touches the object, values of $F_{1}, F_{2}$ and $F_{3}$ are zero. $L_{i}, m_{i}, \theta_{i}, I_{i}, L_{c i}$ and $r_{i}$ are the length, mass, relative rotation angle, moment of inertia, centroid position and pulley radius of the ith link $\operatorname{rod}(i=1,2$ and 3$) . r_{21}$ is the radius of the larger pulley on the middle phalanx and $k_{i}$ is the stiffness of the spring on the ith link rod. $c$ is the system damping coefficient whose value is about 0.01 .

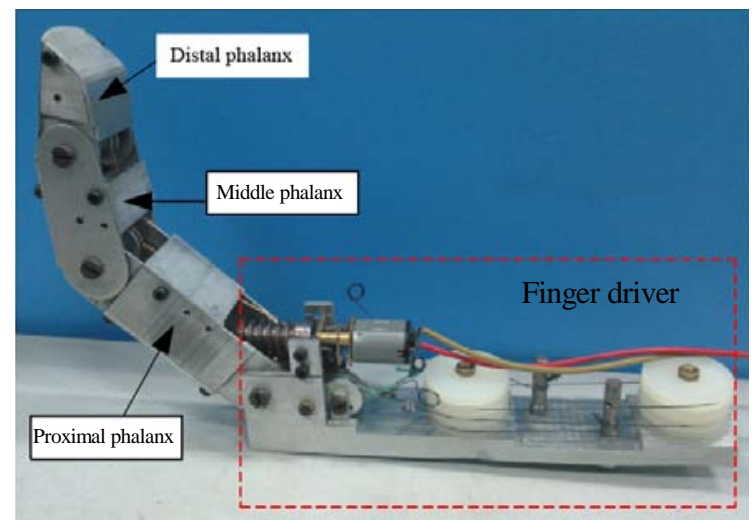

Figure 1. Physical model of the tendon-driven prosthetic finger 
To simplify the modeling process, the models are derived based on the following assumptions: (1) the quality of the tendon is light enough to be ignored compared with other parts of the finger; (2) the tendon rope is rigid, inextensible and in the state of tension all the time with the same tension in different positions; (3) the influence of some nonlinear factors, such as friction, clearance, dead zone, and viscous damping, is not considered in the system; (4) the rod length is not too long, and its weight is very light, so the system gravity potential energy is ignored.

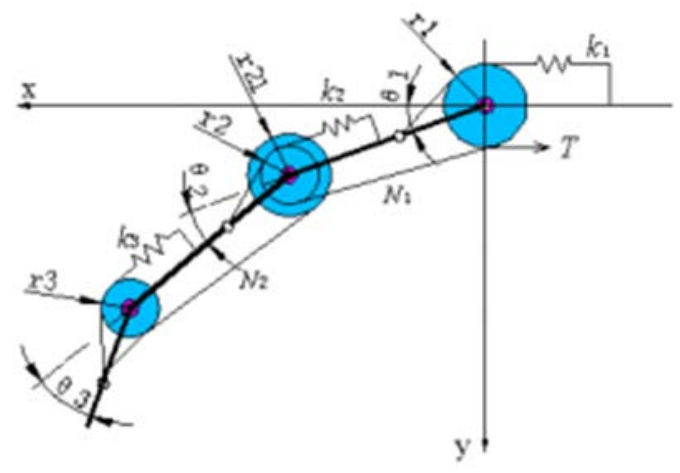

Figure 2. Structure diagram of the tendon-driven prosthetic finger

Select a Descartes coordinate system as shown in Figure 2. $\boldsymbol{X}_{i}=\left[X_{1} Y_{1}\right]^{\prime}(i=1,2$ and 3$)$ denotes the position matrix of the centroid of each link rod. The dynamics model of the prosthetic finger can be obtained through the Lagrange equation [11]:

$$
\tau=D(\theta) \ddot{\theta}+H(\theta, \dot{\theta}) \dot{\theta}+G(\theta)
$$

where $\boldsymbol{\tau}$ is the joint moment matrix, and $\boldsymbol{\tau}=\boldsymbol{T}-\boldsymbol{J F}$. $\boldsymbol{T}$ is the driving torque matrix of joints given by the rope, $\boldsymbol{J}$ is the mapping matrix of external force to the joint torque and $\boldsymbol{F}=\left[F_{1} F_{2} F_{3}\right]^{\prime}$. For the threejoint prosthetic finger, $\boldsymbol{D}(\boldsymbol{\theta})$ is a $3 \times 3$ positive definite symmetric matric, $\boldsymbol{H}(\boldsymbol{\theta}, \dot{\boldsymbol{\theta}})$ is a $3 \times 1$ centrifugation and Coriolis vector and $\boldsymbol{G}(\boldsymbol{\theta})$ is a $3 \times 1$ elastic vector.

Consider the case where only the distal phalanx contacts the object, $F_{1}=F_{2}=0$. The position of the finger at the very beginning of the contact with the object is chosen as the initial position, and initial values of $\theta_{1}, \theta_{2}$ and $\theta_{3}$ are $\theta_{01}, \theta_{02}$ and $\theta_{03}$ respectively. The contact force between the finger and the environment can be simplified as a constant stiffness spring model $[8,9,10]$, that is:

$$
F_{3}=K_{e v} x
$$

where $K_{e v}$ is the environmental stiffness; $x$ is the vertical displacement of the prosthetic finger at the contact point.

The Jacobi matrix of velocity of the proximal phalanx $\left(\boldsymbol{J}_{1}\right)$, which reflects the relationship of the micro-motion in joint space $(\mathrm{d} \theta)$ and the micro-displacement in operating space $\left(\mathrm{d} \boldsymbol{X}_{1}\right)$ of the centroid, is defined as:

$$
J_{1}=\left[\begin{array}{lll}
\frac{\partial X_{1}}{\partial \theta_{1}} & 0 & 0 \\
\frac{\partial Y_{1}}{\partial \theta_{1}} & 0 & 0
\end{array}\right]
$$

Similarly, Jacobi matrices of velocity of the middle phalanx $\left(\boldsymbol{J}_{2}\right)$ and the distal phalanx $\left(\boldsymbol{J}_{3}\right)$ are defined as: 


$$
J_{2}=\left[\begin{array}{ccc}
\frac{\partial X_{2}}{\partial \theta_{2}} & 0 & 0 \\
\frac{\partial Y_{2}}{\partial \theta_{2}} & 0 & 0
\end{array}\right] ; \quad J_{3}=\left[\begin{array}{ccc}
\frac{\partial X_{3}}{\partial \theta_{3}} & 0 & 0 \\
\frac{\partial Y_{3}}{\partial \theta_{3}} & 0 & 0
\end{array}\right]
$$

If the virtual displacement of the prosthetic finger occurs, torque of each joint is $\tau_{i}$ and driving torque given by the rope to each joint is $T_{i}$. Consider the grip balance when only the distal phalanx contacts with the object, force of the distal phalanx is $\boldsymbol{F}_{3}=\left[F_{3 \mathrm{x}} F_{3 \mathrm{y}}\right]$ '. Spring force of link rod 1 and 2 is $\boldsymbol{f}_{1}$ and $\boldsymbol{f}_{2}$ respectively. According to the principle of virtual work [7], the statics equation of the prosthetic finer is derived as:

$$
\boldsymbol{\tau}+\boldsymbol{J}_{1}^{T} \boldsymbol{f}_{1}+\boldsymbol{J}_{2}^{T} \boldsymbol{f}_{2}+\boldsymbol{J}_{3}^{T} \boldsymbol{F}_{3}=0
$$

where $\boldsymbol{\tau}=\left[\begin{array}{lll}\tau_{1} & \tau_{2} & \tau_{3}\end{array}\right]^{\prime}, \boldsymbol{\theta}=\left[\theta_{1} \theta_{2} \theta_{3}\right]^{\prime}$ and $\boldsymbol{X}_{\boldsymbol{i}}=\left[\mathrm{X}_{\boldsymbol{i}} \mathrm{Y}_{\boldsymbol{i}}\right]^{\prime}$.

\section{Force estimation model using motor current signals}

When the prosthetic finger grasps the object stably, the motor and the phalanxes are in the state of force balance, and one has:

$$
T=\tau_{L}=K_{t} i
$$

where $T$ is the motor drive torque, $\tau_{L}$ is the motor load torque, $K_{t}$ is the motor torque constant and $i$ is the motor armature current. When the prosthetic finger grasps the object stably, values of $\theta_{1}, \theta_{2}$ and $\theta_{3}$ are regarded as $\theta_{01}, \theta_{02}$ and $\theta_{03}$ respectively as well, for they change little after the contact. Thus, only $\boldsymbol{F}_{3}$ is unknown. The least square method can be used to solve the above overdetermined equation and calculate the value of $\boldsymbol{F}_{3}$. Taking these values into and using the least square method through a MATLAB program to solve the overdetermined matrix equation, the estimated value of $\boldsymbol{F}_{3}$ can be obtained.

When the prosthetic finger grasps objects with different shapes, values of $\theta_{01}, \theta_{02}$ and $\theta_{03}$ are different. They increase with the decrease of sizes of objects. As values of $\theta_{01}, \theta_{02}$ and $\theta_{03}$ are used to solve the overdetermined equation, the force estimation model is associated with the grasped object. In addition, when the contact positions are different, the value of $S_{3}$ in $\boldsymbol{J}_{3}$ is different. Therefore, the force estimation model is also associated with the contact position. Thus, after solving, the force estimation model can be obtained in the form of:

$$
F_{3}=f\left(i, S_{3}, \theta_{01}, \theta_{02}, \theta_{03}\right)
$$

where $F_{3}$ is the magnitude of vector $\boldsymbol{F}_{3}$.

\section{Grasping control of the tendon-driven prosthetic finger}

\subsection{Grasping force control based on the force estimation model}

A PID controller is chosen to control the grasping force of the prosthetic finger. The PID controller, which is shown in Figure 3, is composed through the linear combination of proportional, integral and differential of the deviation $e(t)=F_{d}(t)-F_{e}(t)$ to control the grasping force, where $F_{d}(t)$ is the desired grasping force and $F_{e}(t)$ is the estimated grasping force by the force estimation model. The mathematical model of the PID controller is: 


$$
u(t)=K_{p} e(t)+K_{i} \int e(t) d t+K_{d} \frac{d e(t)}{d t}
$$

where $K_{p}, K_{i}$ and $K_{d}$ are the proportional, integral and differential coefficients respectively. As the force feedback information uses estimated grasping force based on current signals, not directly force signals, force sensors could be omitted in in practical applications of the control method.

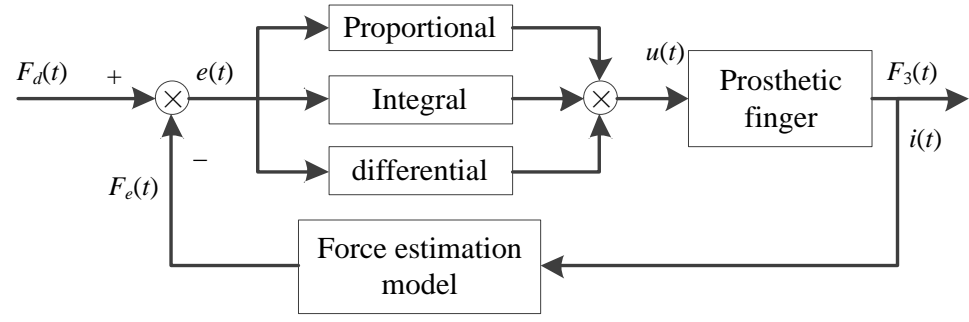

Figure 3. System control block diagram

\subsection{Gasping force control simulation}

The dynamics model, force estimation model, PID controller are simulated combined with a DC motor model in a MATLAB platform. Parameters of the PID controller are obtained as $K_{p}=0.05, K_{i}=$ 0.0005 and $K_{d}=0.01$ by the method of empirical parameter tuning. First, values of $\theta_{01}, \theta_{02}$ and $\theta_{03}$ of three objects with different sizes and shapes are gotten through the numerical solutions of the dynamics equation. Then, give the prosthetic finger a small initial voltage to grasp the three objects with $\theta_{01}, \theta_{02}$ and $\theta_{03}$ as initial positions of each phalanx respectively. The estimated grasping force is used as the feedback information to be compared with the desired grasping force, and the practical grasping force is also recorded in the simulations. Schematic diagrams of grasping objects with different sizes form small to large are shown in Figure 4. The open-loop control performance with the motor voltage being $1 \mathrm{~V}$ is shown in Figure 5.

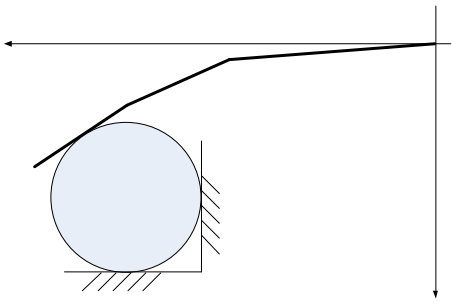

(a) Grasping a large-size object with $\theta_{01}=2.1^{\circ}$, $\theta_{02}=25.1^{\circ}$ and $\theta_{03}=12.5^{\circ}$

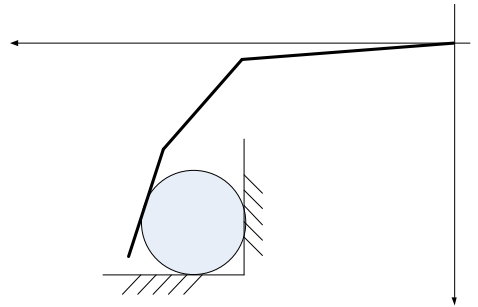

(b) Grasping a middle-size object with $\theta_{01}=3.1^{\circ}$, $\theta_{02}=37.0^{\circ}$ and $\theta_{03}=18.5^{\circ}$

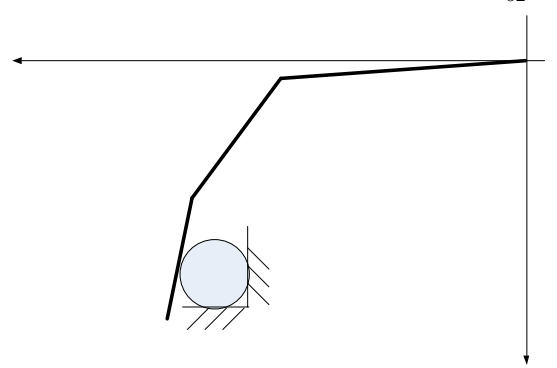

(c) Grasping a small-size object with $\theta_{01}=4.1^{\circ}, \theta_{02}=48.9^{\circ}$ and $\theta_{03}=24.5^{\circ}$

Figure 4. Schematic diagrams of grasping three objects 
It is noticed from Figure 5 that the rising trends of practical and estimated grasping force are not consistent in the initial stage of grasping. This is because the estimation model is derived based on the case of stable grasping, so the estimation is far from being accurate at the very beginning of the contact, so the estimation method does not take effect until the value of the current is more than a certain value. Only the stable values can indicate the accuracy of the force estimation using current signals. It could be calculated form Figure 5 that the relative error of force estimation for grasping these three objects is no more than $15.6 \%$. As we know, when human grasp an object, they do not know the magnitude of the grasping force exactly, so the precision requirement of the force estimation for prosthetic hands is not high, and the relative error of $15.6 \%$ is acceptable. Therefore, the proposed force estimation method is reasonable.

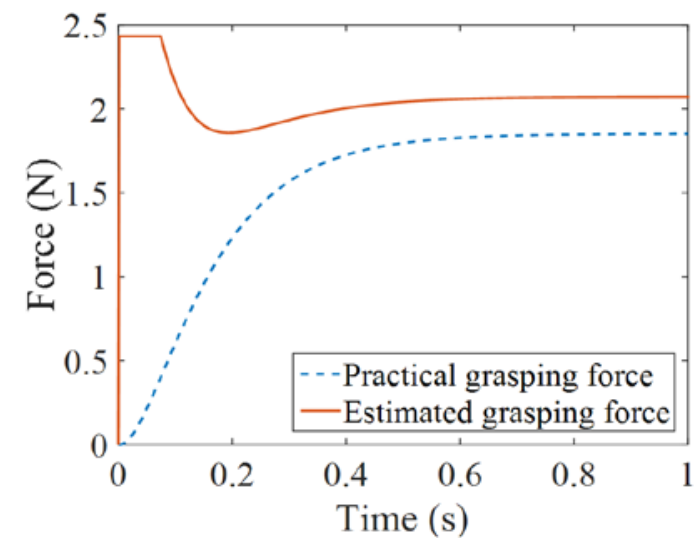

(a) Grasping a large-size object with $\theta_{01}=2.1^{\circ}$, $\theta_{02}=25.1^{\circ}$ and $\theta_{03}=12.5^{\circ}$

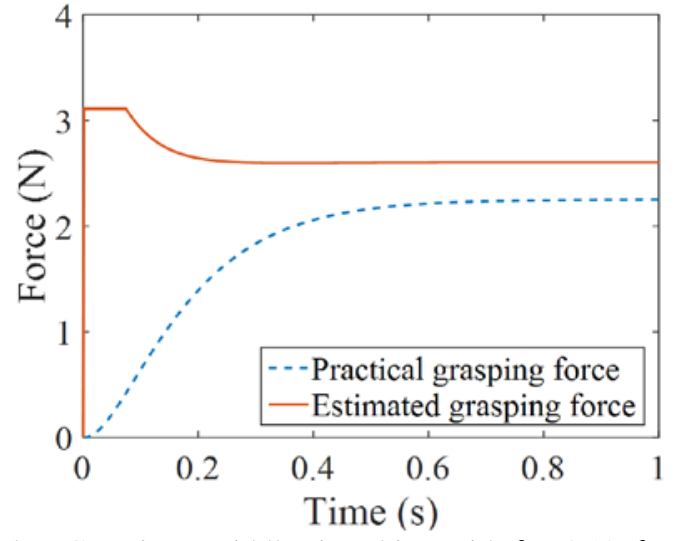

(b) Grasping a middle-size object with $\theta_{01}=3.1^{\circ}, \theta_{02}=$ $37.0^{\circ}$ and $\theta_{03}=18.5^{\circ}$

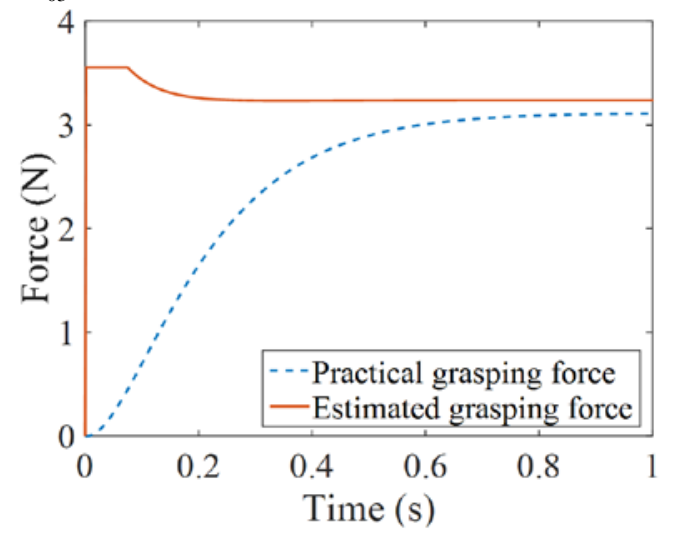

(c) Grasping a small-size object with $\theta_{01}=4.1^{\circ}, \theta_{02}=48.9^{\circ}$ and $\theta_{03}=24.5^{\circ}$

Figure 5. Open-loop control

The closed-loop control performance is shown in Figure 6. As what is done in the open control, the estimated grasping force is also given a relatively small value at the initial stage. It can be seen in Figure 6 that, for all the three objects, the estimated force well follows the desired force, and there is no overshoot of the system response occurring, validating the effectiveness of the PID controller. Similarly, there is a small difference with the relative error also less than $20 \%$ between the estimated force and its practical value in the closed-loop control. 


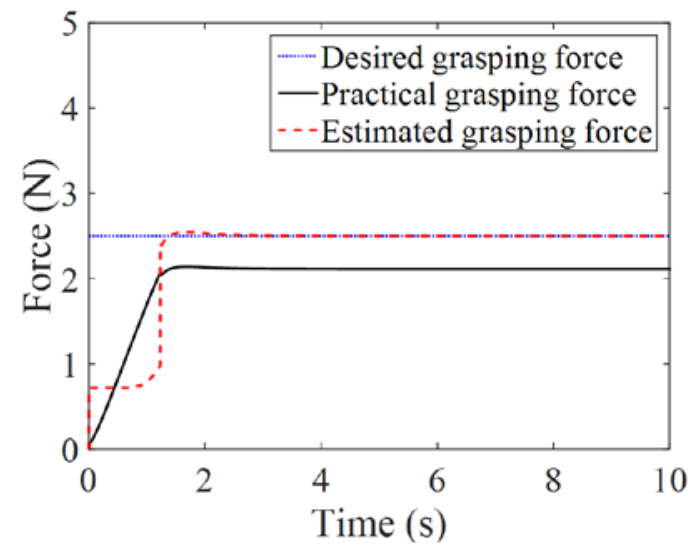

(a) Grasping a large-size object with $\theta_{01}=2.1^{\circ}$, $\theta_{02}=25.1^{\circ}$ and $\theta_{03}=12.5^{\circ}$

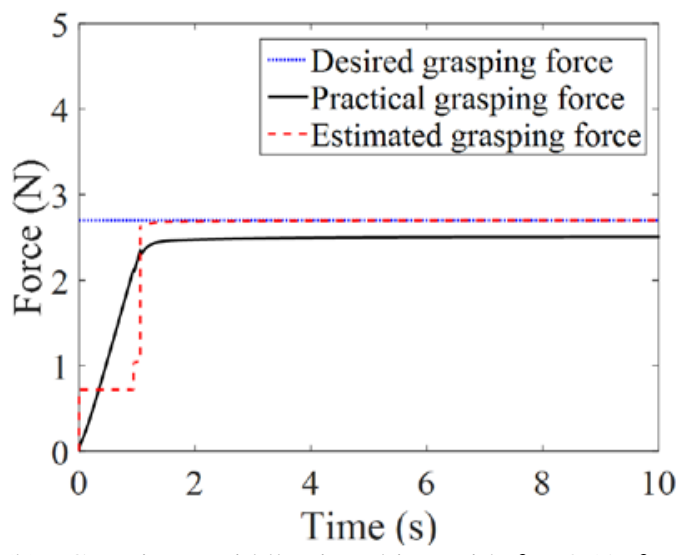

(b) Grasping a middle-size object with $\theta_{01}=3.1^{\circ}, \theta_{02}=$ $37.0^{\circ}$ and $\theta_{03}=18.5^{\circ}$

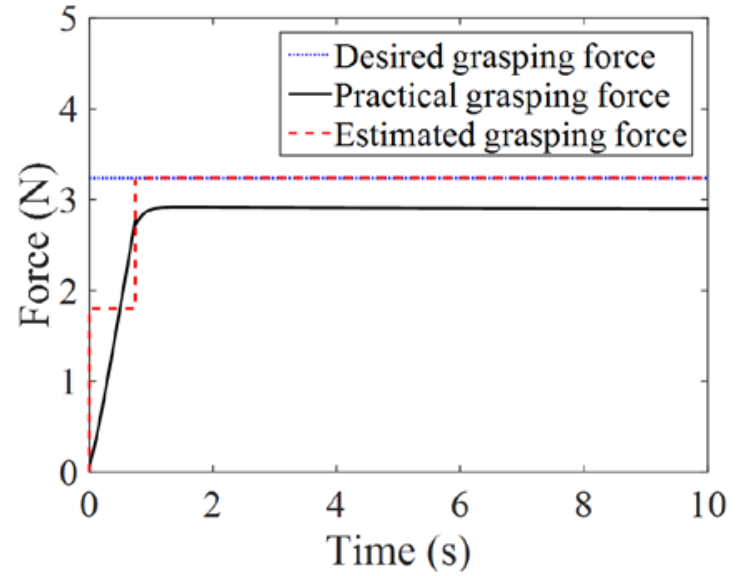

(c) Grasping a small-size object with $\theta_{01}=4.1^{\circ}, \theta_{02}=48.9^{\circ}$ and $\theta_{03}=24.5^{\circ}$

Figure 6. Closed-loop control

The reason for the estimated force value not exactly the same as the actual value is that the force estimation model is a static model and is obtained through the statics analysis of the equilibrium state of grasping, while the simulated value is the result of the dynamics model. Moreover, both the two models are established without the consideration of some nonlinear factors, such as friction, clearance, dead zone and viscous damping, which brings model error. In addition, the estimation method is based on the least square algorithm, and the linearized dynamics model assumes that the grasping force is perpendicular to the distal phalanx, so model gives an approximate estimate of stable grasping force in model, and the specific relationship between the two models and their respective equivalent parameter values need further discussion.

In general, the practical force and the estimated force both follow the desired value with relatively errors less than 20\%. It could also be found from Figure 6 that the force control is stable. Therefore, both the proposed PID grasping force control method and the grasping force estimation method of the prosthetic finger are effective.

\section{Conclusions}

This paper presents a grasping force estimation method using motor current signals for an underactuated tendon-driven prosthetic finger. Then, a PID grasping force control system based on the force estimation model for the prosthetic finger is modeled, where the use of force sensors is avoided. According to the open-loop and closed-loop control simulation results, the proposed estimation and 
control methods are effective. During the control process, the force sensor was substituted by the force estimation model. If the force estimation model was established under different grasping patterns, force sensors could be substituted by the model as frequently as possible. Therefore, the prosthetic hand could be cheaper, lighter and more convenient to use.

\section{Acknowledgment}

This research was supported by the National Basic Research Program of China (973 project: 2011CB013302).

\section{References}

1. R. Clement, K. Bugler, C. Oliver, Surg-Jr. Coll. Surg E 6, 336-340 (2011)

2. R. S. Dahiya, P. Mittendorfer, M. Valle, G. Cheng, V. J. Lumelsky, IEEE Sens. J 13(11), 41214138 (2013)

3. M. C. Carrozza, G. Cappiello, G. Stellin, F. Zaccone, IEEE International Conference on Robotics and Automation 2661-2666 (2005)

4. J. M. Inouye, F. J. Valero-Cuevas, Int. J. Robot Res 33(5), 694-705 (2014)

5. F. Lotti, P. Tiezzi, G. Vassura, L. Biagiotti, G. Palli, C. Melchiorri, International Conference on Robotics \& Automation 2005, 4488-4493 (2005)

6. L.Zollo, S. Roccella, E. Guglielmelli, M. C. Carrozza, P. Dario. Biomechatronic, IEEE/ASME Transactions on Mechatronics 12(4), 418-429 (2007)

7. N. E. Joukowski, Theoretical mechanics (Springer, Berlin Heidelberg, 2010)

8. D. Erickson, M. Weber, I. Sharf, Int. J. Robot. Res 22(1), 41-58 (2003)

9. G. Gilardi, I. Sharf, Mech. Mach. Theory 37(10), 1213-1239 (2002)

10. J. Park, O. A. Khatib, Int. J. Robot. Res 25(25), 575-591 (2006)

11. D. Zhang, Z. Wang, IEEE 299 - 304 (2013) 